\title{
La formación del Talento Humano y las Competencias Profesionales: Un nexo indisoluble para el éxito laboral.
}

(c) (1)(2)(2)

The training of Human Talent and Professional Skills: An indissoluble link for job success.

Norma Yolanda Villacis Venegas. ${ }^{1}$

Recibido: 12-06-2021 / Revisado: 22-06-2021 /Aceptado: 10-07-2021/ Publicado: 05-08-2021

\begin{abstract}
.
DOI: https://doi.org/10.33262/concienciadigital.v4i3.1.1812

Introduction. Opting for a training model that results in a more competent, innovative and comprehensive professional and that contributes to job success, implies changes in the didactic-methodological strategies that are developed at the university, which is not always easy. Target. Objective. Establish the importance of the relationship between the training process of human talent and the development of skills in professionals, as an indissoluble link for job success. Methodology. A descriptive, non-experimental methodology was used, based on the documentary review and theoretical methods, under established inclusion criteria. Results. 1 . The initial idea of the research was confirmed: There is an indissoluble link between the training process and the professional competencies achieved by each student; The more optimization is achieved in the process of training human talents, the better performance and professional competencies they will achieve, which positively influences individual job success and also the organizations and companies where new professionals are inserted. 2. It is necessary to implement and develop new didactic-methodological strategies to provoke a meaningful teachinglearning process that allows contributing to the training of competent, innovative and
\end{abstract}

\footnotetext{
${ }^{1}$ Escuela Superior Politecnica de Chimborazo Sede Morona Santiago, Carrera de Contabilidad y Auditoría, norma.villacis@espoch.edu.ec, https://orcid.org/0000-0002-6314-3805
} 
comprehensive professionals and to quality in job placement, satisfying social demands and expectations and labor. 3. Guidelines are established that may constitute possible solutions. Conclusions. Optimization in the training and curricular process requires that it be streamlined, together with the implementation of novel methodological strategies, on scientifically sound bases. This process is closely linked to the development of professional competences, which requires having a committed and updated faculty, capable of migrating from a Traditional Didactics to a Contemporary Didactics and that can establish in the teaching-learning process the indissoluble link between : training processes, development of professional skills and job success.

Keywords: Human Talent, professional skills, job success, training, teaching-learning process.

\section{Resumen.}

Introducción. Optar por un modelo de formación que logre como resultado un profesional más competente, innovador e integral y que tribute al éxito laboral, implica cambios en las estrategias didácticas-metodológicas que se desarrollan en la universidad, lo cual no siempre es sencillo. Objetivo. Fundamentar la importancia de la relación entre el proceso formativo del talento humano y el desarrollo de competencias en los profesionales, como nexo indisoluble para el éxito laboral. Metodología. Se empleó una metodología descriptiva, no experimental, con base en la revisión documental y en métodos teóricos, bajo criterios de inclusión establecidos. Resultados.1.Quedó confirmada la idea inicial de la investigación: Existe un nexo indisoluble entre el proceso formativo y las competencias profesionales alcanzadas por cada estudiante; mientras mayor optimización se logre en el proceso de formación de los talentos humanos, mejor desempeño y competencias profesionales alcanzarán, lo cual influye de manera positiva en el éxito laboral individual y también de las organizaciones y empresas donde se inserten los nuevos profesionales. 2. Es necesario la implementación y desarrollo de nuevas estrategias didácticas-metodológicas para provocar un proceso de enseñanzaaprendizaje significativo que permita contribuir a la formación de profesionales competentes, innovadores e integrales y a la calidad en la inserción laboral, satisfaciendo las demandas y expectativas sociales y laborales. 3. Se establecen orientaciones que pueden llegar a constituir posibles soluciones. Conclusiones. La optimización en el proceso formativo y curricular exige que este sea dinamizado, unido a la implementación de estrategias metodológicas novedosas, sobre bases científicamente sólidas. Dicho proceso está íntimamente ligado al desarrollo de las competencias profesionales, lo cual requiere contar con un claustro comprometido y actualizado, capaz de migrar de una Didáctica Tradicional, hacia una Didáctica Contemporánea y que pueda establecer en el proceso de enseñanza-aprendizaje el nexo indisoluble entre: procesos formativos, desarrollo de competencias profesionales y éxito laboral.

Palabras clave: Talento Humano, competencias profesionales, éxito laboral, formación, proceso de enseñanza-aprendizaje. 


\section{Introducción.}

La época moderna marcada por los ostensibles cambios producidos por la globalización y el desarrollo científico -tecnológico alcanzado en las diferentes esferas de actuación, han dado origen al empleo de nuevas estragias didácticas - metodológicas en los procesos formativos y curriculares. Establecer la relación entre formación del talento humano y el perfeccionamiento de las competencias profesionales en los egresados, sigue constituyendo un aspecto esencial para lograr un mejor desempeño y por consiguiente el éxito laboral.

En este orden es importante comprender que, el proceso de formación de los talentos humanos ha venido transitando por diferentes etapas, las cuales están íntimamente ligadas con el propio desarrollo histórico - social y educativo. Lo anterior provoca pensar en la relación que se da entre las categorías educación y desarrollo, como componentes esenciales del proceso formativo.

Según Agudelo, (2018), en la era actual, denominada: del conocimiento y la innovación, las organizaciones cumplen un papel primordial, pero mucho más importante es el lugar que ocupan las personas que las integran; precisamente porque son estas quienes dinamizan todo el proceso de apertura a nuevos conocimientos y aprendizajes, influyendo proporcionalmente en la competitividad y sostenibilidad empresarial y organizacional.

En correspondencia a lo explicado cabe preguntarnos ¿Cómo se puede lograr optimizar el proceso formativo de los talentos humanos para que este sea propulsor del desarrollo de competencias profesionales en los egresados de las diferentes áreas del saber y por tanto conducentes al éxito laboral? Considerando lo planteado, el objetivo del trabajo se define en la necesidad de: Fundamentar la importancia de la relación entre el proceso formativo del talento humano y el desarrollo de competencias en los profesionales, como nexo indisoluble para el éxito laboral.

El proceso formativo del Talento Humano, las prácticas preprofesionales y el desarrollo de competencias profesionales: Condicionantes para el éxito laboral.

Según el Diccionario de la Real Academia Española (2020), el término Talento Humano proviene de lat. talentum 'moneda de cuenta', 'unidad de peso', y este

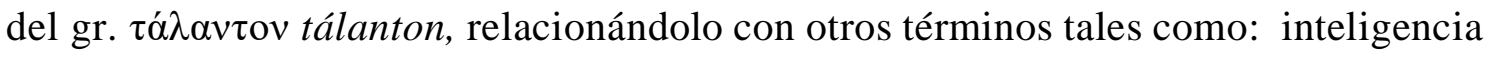
(capacidad de entender y con aptitud, definiendo esta como la capacidad para el desempeño de algo). Resumiendo en una primera acepción: se trata de la persona inteligente o apta para determinada ocupación.

Comprender el significado de este término requiere considerar otras posibles acepciones o definiciones resultantes de investigaciones relacionadas con este tema. Por ejemplo: Moreno \& Godoy, (2012), señalan que es un término que hay que apreciarlo desde diferentes contextos, uno sería el contexto organizacional, donde se utilizan indistintamente dos acepciones: recursos y talento, entendiendo que los recursos humanos están representados por el universo de la organización, mientras que el talento humano 
está caracterizado por la individualidad y diversidad de profesiones u ocupaciones, a quienes se les determinan sus competencias (conocimientos, habilidades, destrezas) y por tanto identificar el potencial individual que poseen (p. 61).

En el caso del trabajo que se presenta relacionamos el término "Talento Humano", con aquellas personas que dado el nivel de competencias, conocimientos, aptitudes, habilidades y destrezas logran adquirir de forma individual durante el proceso formativo y curricular en las aulas universitarias y dado esto pueden insertarse en el mercado laboral y contribuir al éxito de la empresa u organización. En este sentido especial interés tiene establecer la relación que se da entre el proceso formativo y el desarrollo de competencias profesionales de los egresados de las diferentes carreras.

En consonancia con lo señalado es importante dejar claro que los modelos educativos que actualmente se despliegan en las diferentes universidades deben procurar que el proceso de enseñanza-aprendizaje sea cada vez más significativo para los estudiantes, de modo tal que favorezca el desarrollo de sus competencias genéricas y específicas. Se coincide con Recalde \& Maqueira, (2017), al plantear que mientras más diversos y adaptables sean los modelos curriculares, mejor respuesta se logrará en los procesos formativos, es decir, dependiendo de la calidad y diversidad del proceso formativo, así será el desarrollo de competencias de los profesionales que egresan de las aulas universitarias independientemente a su condición.

Exclusiva utilidad para el tema de investigación tiene los criterios de Restrepo, (2016), al señalar que es precisamente: "El talento humano el factor diferenciador y el activo más valioso de cualquier organización u empresa". Reflexionando sobre la opinión de la autora se participa en que el nivel de competitividad y éxito de una empresa está directamente ligado al perfeccionamiento que debe darse en los procesos formativos de los talentos humanos que posteriormente integraran dicha organización u empresa.

Se converge con Restrepo, (2016), al comentar que: "los procesos de formación curricular de los futuros egresados de las diferentes carreras deben estar alineados con la estrategia de la organización, con sus valores, su misión, visión y con su cultura". Apunta la autora con mucha certeza, que dichos procesos de formación, tienen entre sus propósitos la transferencia de conocimientos, tendientes a dinamizar la cultura organizacional para facilitar así el logro de los objetivos estratégicos de la empresa u organización.

En este orden es fundamental aplicar el principio de la Pedagogía General, referente al vínculo de la teoría con la práctica, como condición indispensable para el logro de un aprendizaje significativo para la vida. Es por ello que una de las aristas a atender y desarrollar durante todo el proceso formativo es la práctica preprofesional. En este entramado de ideas Crebert et al, (2004), señaló que las prácticas preprofesionales pueden ayudar a los estudiantes a desarrollar habilidades interpersonales que no se consiguen en un salón de clases.

Por su parte Guarnizo, (2018), explica que las prácticas preprofesionales son una alternativa de oportunidad laboral que recibe el estudiante ante uno de los principales 
fines de formación en una Institución de Educación Superior. En ese mismo año, De la Flor, (2018), señala que las prácticas preprofesionales están diseñadas para construir capital humano y mejorar la adquisición de habilidades.

Lo cierto es que la rotación de los estudiantes durante su formación curricular por diferentes entidades, empresas u organizaciones los proveerá progresivamente del desarrollo de aquellas competencias específicas para el área donde se está formando. Por otra parte les permitirá ir adentrándose paulatinamente en el ambiente laboral, reconociendo en este las formas de interacción, los objetivos, procedimientos de trabajo y funcionabilidad de la organización. Todo esto constituyen condicionantes esenciales para propiciar el éxito laboral tanto de forma individual como colectiva.

\section{¿Por qué se debe dar un nexo indisoluble entre proceso formativo, las competencias profesionales y éxito laboral?}

Diversos son los investigadores que han aportado al tema del proceso formativo y su impacto en el desarrollo de las competencias profesionales y su interrelación con el éxito laboral. En este orden Amaru, (2009), precedida de los aportes de otros importantes investigadores, explica que para tener éxito, las organizaciones deben lidiar con el cambio continuo y convertirse en organizaciones en aprendizaje.

Lo anterior adquiere vital transcendencia para la investigación que compartimos, precisamente porque responde a la necesidad de establecer el nexo entre estos tres procesos: la formación, el desarrollo de competencias profesionales y el éxito laboral. En sintonía con lo explicado concordamos con Bravo; et al (2016), al destacar que cada vez es más importante establecer la interrelación entre los talentos y recursos humanos con la gestión del conocimiento, la innovación y la responsabilidad social empresarial. Subraya el autor que este tema dada su significación aparece como una línea investigativa de gran efecto que puede aportar a la integración de estos factores y a establecer la aplicación de un enfoque sistémico que ayude a conceptualizar el papel de la gestión del talento humano en la empresa actual.

En esta misma línea de pensamiento Saldarriaga Ríos (2013), enfatiza en la necesidad de alcanzar una integración que facilite que la gestión de recursos humanos tribute a la gestión de los procesos de conocimiento, desde su creación hasta su aplicación. Según el autor, esto contribuirá a mejorar los resultandos, así como al incremento de la productividad y la competitividad individual y empresarial, favoreciendo de esta forma el perfeccionamiento de la responsabilidad social y de la calidad de vida del talento humano que ah egresado y se inserta al mercado laboral; de ahí que sea una prioridad dentro de las estrategias de gestión humana a dar seguimiento y atención permanente.

Salanova, (2009), seguido de Armas et al (2017), sostienen que "las organizaciones modernas están cambiando en una dirección que se basa cada vez más en el conocimiento intelectual y psicológico, la experiencia y el talento; así como, en la autogestión y la atención a necesidades individuales y colectivas de los empleados, de la organización y de la sociedad en general" . En esta línea se reconoce la importancia de que el proceso 
formativo debe ser integral, permitiendo al egresado no solo desarrollar sus competencias profesionales, sino también tener una inserción laboral de calidad, manteniendo su integralidad física y psicológica.

Como puede apreciarse todo lo abordado encierra un gran valor científico, específicamente lo concerniente a los modelos formativos y curriculares y su correlación con el desarrollo de competencias profesionales como condición previa para garantizar el éxito laboral. Somos del criterio que mientras más completo sea este proceso, mejor preparado y competente serán los egresados. Es por esta razón que optar por un modelo de formación que logre como resultado un profesional más competente, innovador e integral y que a su vez tribute al éxito laboral individual y colectivo, implica cambios en las estrategias didácticas-metodológicas que se desarrollan en las aulas universitarias, lo cual no siempre es sencillo de lograr.

Alcanzar lo anterior requiere del cumplimiento de diferentes directrices y políticas; dentro de estas es necesario contar con un sistema de capacitación permanente dirigido a todos los profesionales que intervienen en el proceso formativo y curricular; además es necesario tener un claustro motivado, innovador, comprometido y actualizado, capaz de provocar un proceso de enseñanza -aprendizaje significativo, basado entre otros aspectos en la investigación y en el empleo de las Nuevas Tecnologías de la Información y Comunicación, que pueda migrar de una Didáctica Tradicional, hacia una Didáctica Contemporánea.

En articulación con lo explicado se considera importante establecer el nexo entre el proceso formativo, el desarrollo de competencias profesionales y el éxito laboral a alcanzar tanto de forma individual como organizacional o empresarial y es ahí precisamente donde está el reto actual en los procesos formativos en las diferentes carreras y universidades. Encontrar un equilibrio que permita el desarrollo ascendente y sostenible en ello seguirá siendo un gran desafío, pero necesariamente debe ser resuelto desde la perspectiva de formar un talento humano más competente e integral.

\section{Metodología.}

Para la investigación se empleó una metodología descriptiva, no experimental, con base en la revisión de la bibliografía 'precedente y en métodos teóricos como: el históricológico, el analítico - sintético, inductivo - deductivo y la revisión documental; los cuales favorecieron la búsqueda sobre los componentes fundamentales del problema objeto de estudio. Los conectores claves para el desarrollo de la investigación se establecieron los siguientes: talento humano, competencias profesionales y éxito laboral.

Criterios de Inclusión

1. Tipo de estudios: Estudios de revisiones teóricas o experimentales que hayan sistematizado o desarrollado teorías en relación con el tema objeto de estudio.

2. Tipo de participantes: investigadores, docentes, profesionales del área.

3. Tipo de resultados: Revisiones Bibliográficas, Estudios Descriptivos, 
Documentales, Metodologías, Programas, Estrategias de intervención relacionadas con el tema de investigación.

Criterios de exclusión.

1. Otros estudios que por su tema o resultados no aporten elementos significativos para la investigación.

La calidad se estableció mediante el seguimiento de los criterios de inclusión y exclusión instaurados previamente. Para la búsqueda de los antecedentes primarios de investigación se utilizaron buscadores de información científica especializada, tales como: Google Académico, Latindex, Dialnet, Scopus, específicamente artículos y libros relacionados con el tema.

\section{Resultados.}

La Revisión de la literatura precedente permite establecer como principales resultados los siguientes:

1. Quedó confirmada la idea inicial de la investigación: Existe un nexo indisoluble entre el proceso formativo, las competencias profesionales alcanzadas por cada egresado y el éxito laboral; mientras mayor optimización se logre en el proceso de formación de los talentos humanos, mejor desempeño y competencias profesionales alcanzarán, lo cual influye de manera positiva en el éxito laboral individual y también de las organizaciones y empresas donde se inserten los nuevos profesionales. Ello nos hace coincidir con Saldarriaga Ríos (2013), Bravo; et al (2016), Restrepo, (2016), al enfatizar en la necesidad de dinamizar los procesos formativos, de manera tal que respondan a las exigencias actuales y a las demandas de las diferentes organizaciones y empresas.

2. Es necesario la implementación y desarrollo de nuevas estrategias didácticasmetodológicas que permitan provocar un proceso de enseñanza-aprendizaje altamente significativo, para poder contribuir objetivamente a la formación de profesionales competentes, innovadores e integrales y favorecer la inserción laboral con profesionales de calidad que cumplan con las demandas y expectativas sociales y laborales. Concordándose con los aportes de Salanova, (2009), seguido de Armas et al (2017), los cuales destacan la importancia de la formación integral como componente esencial para el éxito empresarial.

3. Derivado del análisis realizado se establece un sistema de orientaciones que de implementarse pueden llegar a constituir posibles soluciones para la optimización del proceso formativo y el desarrollo de competencias profesionales que permitan a los futuros egresados contribuir al éxito laboral y organizacional. Destacan en estas las siguientes: 
4. Se debe dinamizar el proceso formativo y curricular, de modo tal que se integre en el proceso de enseñanza-aprendizaje el desarrollo de competencias profesionales que permitan no sólo el aprendizaje de la técnica específica en las diferentes carreras, sino también el desarrollo y fortalecimiento de aquellas competencias que favorezcan un mejor desempeño de las cualidades humanas, éticas y morales.

5. La formación debe estar íntimamente ligada al cumplimiento de los Cuatro Pilares declarados por la Delors, (1994), presidenta de la UNESCO: Saber Conocer, Saber Hacer, Saber Convivir, Saber Ser, como única alternativa para lograr la formación integral de los egresados y el desarrollo de aprendizajes significativos para la vida.

6. El proceso formativo debe caracterizarse por la vinculación de la teoría con la práctica; ello contribuye a que sea altamente significativo y a su vez facilita el dominio del sistema de conocimientos, técnicas, habilidades y competencias inherentes al perfil profesional de cada carrera y al vínculo de este con la práctica.

7. Debe procurarse además que el proceso formativo evalué de manera sistemática el cumplimiento y sostenibilidad de la oferta académica, de forma tal que facilite el nexo entre el proceso de enseñanza-aprendizaje y los objetivos y alcances de las diferentes organizaciones y empresas donde se insertaran los egresados. Es por ello fundamental dar seguimiento a la práctica preprofesional como sustento para el desarrollo de competencias prácticas en los futuros profesionales.

8. Para garantizar la calidad del proceso formativo y el desarrollo de competencias profesionales en los egresados se debe contar con un sistema de capacitación permanente, el cual provea a los docentes de un nivel de actualización sistemático, permitiéndole perfeccionar las estrategias didácticas metodológicas que empelan en el proceso de enseñanza-aprendizaje.

9. Como parte del proceso formativo debe lograrse además el empoderamiento de los estudiantes por alcanzar mejores resultados, desarrollando en ellos el compromiso por ser cada día mejores y más competentes. Se les debe enseñar a medir sus competencias, a reconocer sus potencialidades, pero también, sus debilidades y algo fundamental orientarlos para que encuentren soluciones efectivas y puedan convertir las limitaciones en fortalezas.

10. De igual manera es muy importante que aprendan a mejorar su calidad de vida mediante la optimización de sus competencias y su contribución al desarrollo del bien colectivo y a la obtención de resultados que tengan impacto y permitan la transformación de la realidad.

11. Por último resulta imprescindible garantizar el nexo del proceso formativo con los alcances y competencias a lograr en los egresados. El proceso de enseñanzaaprendizaje debe optimizarse facilitando el desarrollo de competencias profesionales que permitan a los futuros egresados contribuir al éxito laboral y organizacional. 


\section{Conclusiones.}

- La optimización en el proceso formativo y curricular, exige la implementación de estrategias didácticas -metodológicas novedosas y significativas, erigidas sobre bases científicamente sólidas. Dicho proceso está íntimamente ligado al desarrollo de competencias profesionales, resultando una condicionante esencial para el logro del éxito laboral individual o colectivo en cualquier empresa o entidad. Un profesional competente, será siempre esencial para el éxito laboral de cualquier empresa o entidad.

- Para formar profesionales más competentes e integrales hoy más que nunca es necesario dinamizar los procesos formativos y curriculares, para ello se requiere entre otros aspectos contar con un claustro comprometido y actualizado capaz de migrar de una Didáctica Tradicional, hacia una Didáctica Contemporánea, de forma tal que en el proceso de enseñanza-aprendizaje se logre establecer el nexo indisoluble existente entre: procesos formativos, desarrollo de competencias profesionales y éxito laboral.

\section{Referencias Bibliográficas.}

Agudelo, BE., (2018), Formación del talento humano y la estrategia organizacional en empresas de Colombia. Ciencias Sociales. Entramado. Enero - Junio, 2019 vol. 15, no. 1, p. 116-137 http://dx.doi.org/ 0.18041/1900-3803/entramado.1.5383 DOI: https://doi.org/10.18041/1900-3803/entramado.1.5383.

Amaru, A. (2009). Fundamentos de la Administración: Teoría General y Proceso Administrativo. México: Pearson- Prentice Hall.

Armas, YM., Llanos, MP., Traverso, PA., (2017) Gestión del Talento Humano y Nuevos Escenarios Laborales. Edición ECOTEC ISBN 978-9942-960-29- No Pág 139. Samborondón - Ecuador

Bravo, WA., Delgado, BI., Parrales, CG., (2016) Análisis de las investigaciones sobre Talento Humano: Revista Publicando, págs. 354-365.

Crebert, G., Bates, M, Bell, B., Patrick, CJ., \& Cragnolini V., (2004). Developing generic skills at university, during work placement and in employment: graduates' perceptions. Higher Education Research \& Development, 23(2), 147-169.

Diccionario de la Lengua Española. Edición Tricentenario. Actualización 2020. https://dle.rae.es/

Delors, J., (1994). Los cuatro pilares de la educación, en La Educación encierran un tesoro. México: El Correo de la UNESCO, pp. 91-103.

De la Flor, L., (2018) La importancia de las prácticas preprofesionales en la transición al empleo Un estudio en las ciudades capitales del Perú 
Guarnizo, SF., (2018) Importancia de las prácticas pre profesionales para los estudiantes de educación superior en la Universidad de Guayaquil. Revista INNOVA Research Journal, Vol. 3, No.8 pp. 14-25 DOI: https://doi.org/10.33890/innova.v3.n8.2018.717 URL: http://revistas.uide.edu.ec/index.php/innova/index

Moreno, F., et al. Godoy, E. (2012). El talento humano: Un capital intangible que otorga valor en las organizaciones (human talent: An intangible capital that gives value in organizations). Daena: International Journal of Good Conscience, 7(1), 57-67.

Recalde, A., \& Maqueira, G., (2017), El Modelo Educativo de la Universidad de Guayaquil. Una experiencia inclusiva Lecturas: Educación Física y Deportes, Revista Digital. Buenos Aires http://www.efdeportes.com/ Año 21, № 225,

Restrepo, A (2016). Los procesos de formación del talento humano como factor de desarrollo organizacional. https://rhpositivo.net > procesos-de-formacion.

Salanova. (2009). Organizaciones saludables, Organizaciones Resiliencias.

Saldarriaga, J. G. (2013). Responsabilidad social y gestión del conocimiento como estrategias de gestión humana. Estudios Gerenciales, 29(126), 110-117. DOI: http://dx.doi.org/10.1016/S0123-5923(13)70026-3.

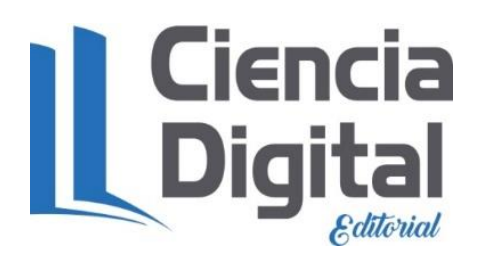




\section{PARA CITAR EL ARTÍCULO INDEXADO.}

Villacis Venegas, N. Y. (2021). La formación del Talento Humano y las Competencias Profesionales: Un nexo indisoluble para el éxito laboral . ConcienciaDigital, 4(3.1), 6675. https://doi.org/10.33262/concienciadigital.v4i3.1.1812

\section{¿Ciencia}

El artículo que se publica es de exclusiva responsabilidad de los autores y no necesariamente reflejan el pensamiento de la Revista Conciencia Digital.

El artículo queda en propiedad de la revista y, por tanto, su publicación parcial y/o total en otro medio tiene que ser autorizado por el director de la Revista Conciencia Digital.

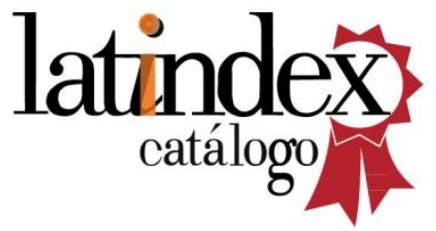

